\title{
Framing Antecedents of the Last Mile Omni-Channel Distribution for the Retail Apparel Group
}

\author{
Dr Thokozani Patmond Mbhele \\ Sanjana Rambaran \\ Discipline of Supply Chain Management, School of MIG, University of KwaZulu-Natal \\ (Westville Campus) \\ Private Bag X54001, Durban, 4000, KZN Province, South Africa, Tel. no: +2731 260 7524, \\ E-mail: mbhelet@,ukzn.ac.za
}

\begin{abstract}
The last mile has to deliver the goods at the right time and at the right cost for business logistics services to enhance the customer service experience. The objective of this paper is to examine the challenges of retail omni-channel distribution influencing the effective last mile fulfilment operations processes in the apparel group (organisation phase). It further establishes the extent of how the last mile for demanddriven store replenishment influences the level of retailers' order fulfilment (in full and on time) (environment phase). This paper, persistently assesses the contribution of integrated information systems on brick and mortar and virtual omni-channel distribution networks to improve timeousness, order accuracy and fill rate (technology phase). The mixed method has triangulated the methods from both quantitative methodology using frequencies and binomial testing and subjective thematic analysis of qualitative data from interviewees. The sample of 175 questionnaires were received with a response rate is 85 per cent (148 responses). The main findings of the paper revealed that the current order fulfilment process aligned with the last mile is effective on the basis of the fill rate, lead time and order condition of orders delivered via store economy and store express.
\end{abstract}

Key Words: Last mile, omni-channel distribution, TOE framework, order fulfilment,

\section{Introduction}

The logistics hubs have played an important role in traditional delivery solutions, but the application of Industry 4.0 inventions has made it possible to redefine the hub and spoke of a centralised transport topology optimisation paradigm. If the first mile is a core component of electronic commerce (ecommerce) on inbound and outbound warehousing and value-added services, the last mile is the final link to deliver the goods at the right time and at the right cost for business logistics service and for customer service experience. In this case, the first mile operations (pickups) and the last mile operations (deliveries) should be integrated to optimise the operation costs and the utilisation of resources, like package delivery trucks, drivers, and hubs. The integrated model include the assignment of first mile and last mile delivery tasks to the available resources and the optimisation of operation costs, while constraints like capacity, time window, and availability are taken into consideration (Bányai , Illés, and Bányai, 2018:15).The research problem resonates with how the integrated first mile and last mile provide a flexi delivery modus operandi whereby customers should increasingly expect to indulge and impress on when, where, and how products and/or services are delivered for a profound service experience, irrespective of spikes and seasonal logistics. The main challenges of last mile resonate with infrastructural concerns regarding transportation structure, information and knowledge sharing, and advanced technology; customer issues such as incorrect addresses, unavailability at home and cancellation; fuel and time wastage as excessive expenditure; and product types such as temperaturesensitive, flammable, toxic, or fragile, which require businesses to plan the last-mile transportation accordingly. The retailers still find digital channels and channel integration as posing a challenge on inventory management and distribution with the need synchronised between first mile and last mile 
logistics dimensions (Piotrowicz and Cuthberton, 2014:2). The main aim cogitates and examines the challenges of retail omni-channels distribution influencing the effective last mile fulfilment operations processes in the apparel group. Beck and Rygl (2015:170) argue that the landscape of fragmented channels affect the efficiency of service experience; firstly, online order and collect in-store depend on the customer's device or location, secondly, searching for information in the store and on the mobile device for attractive offers and enticing prices, and finally, seeking information online and buying offline as webrooming, or purchase in another channel (Verhoef, Kannan and Inman, 2015).

\section{Nature of South African Market}

The growth of electronic commerce (e-commerce) in South Africa is dependent on value-based quality of Internet services, and the reliability and cost of delivery mechanisms such as first mile and the last mile (translating into the placement of the order, payment, and the delivery). The last mile logistics within South Africa are slightly different in the level of innovation as more companies benefit from scale economies due to growth and optimised logistics. Firstly, vertical integration: large online retailer Takealot has purchased Mr Delivery and vertically integrated their logistics including warehousing and delivery. Secondly, store distribution networks: others such as Makro and Pick n Pay leverage off their store distribution network, warehouse, and delivery hubs (though it can be noted that Pick n Pay and Woolworths have also created warehouses and distribution for e-commerce alone). They may also use their stores as collection points (Beck and Rygl, 2015). Thirdly, outsourced logistics: other online retailers often make use of courier optimising companies such as uAfrica and Parcelninja which provides assistance in logistics optimising, using other courier companies. Parcelninja as an ecommerce fulfilment specialist provides scalable outsourced e-commerce warehousing and fulfilment for small and large online business. These companies may also provide warehousing and fulfilment capabilities. Alternatively couriers collect parcels from manufacturers or sellers and deliver to final consumers without warehousing required. Although new delivery approaches, such as stores hiring their own delivery personnel, and start-ups outsourcing delivery vehicles and drivers who can operate effectively only on a very local basis. Uber eats, and fast-food outlet-based delivery systems gain few advantages by building scale geographically in the apparent industry. In South Africa retail stores that have online channels include sites such as Makro, Woolworths, Mr Price, Foschini group stores, Truworths group stores using online order and deliver channels (Verhoef, Kannan and Inman, 2015), but may include hybrid options such as 'click and collect' in which shopping can be done online and collected in retail stores, or the facilitation of returns to offline stores. The authors further describe the omni-channel management as the synergetic management of the numerous available channels and customer touchpoints, in such a way that the customer experience across channels and the performance over channels is optimised and in return, maximise the firm's value (Verhoef, Kannan and Inman, 2015; Hure, Picot-Coupey and Ackermann, 2017). The interplay between channels and brands (Verhoef et al., 2015) entrenches the sync within the brand ecosystem on consumers that reinforces consumer loyalty.

\section{Last Mile on Omni-Channel Delivery}

Onstein, Tavasszy and Damme (2019:244) provide the distinct structures that "centralised structures may include a single distribution centre (DC) location or, sometimes, direct shipment is used". Spree.co.za, Woolworths and Mr Price use direct shipment to transport products to their private customers as the last mile for omni-channel utilisation. The authors further describe "decentralised distribution structures to include multiple DC locations in a so-called multi-echelon system. Fashion shipper Zara recently decided to further decentralise distribution by adding a new DC in the Netherlands (op de Woerd, 2017), including online shipper Amazon investing heavily on a decentralised structure. Boateng (2014), Beck and Rygl (2015) and Verhoef et al., (2015) refer to distribution in multiple 
channels as omni-channel distribution, whereby retailers may decide to integrate operations of order fulfilment, inventory availability and positioning and efficient distribution, and physically synchronised product flows in order to provide a seamless shopping experience (Hubner, Holzapfel and Kuhn, 2016). Omni-distribution is "the fulfilment of customer orders from a myriad of channels integrated by one system with visibility of the location of the item and delivery date at a price that is acceptable to the customer and profitable by the retailer" (Mcbeathe, 2014:2). Nevertheless, decentralised DC systems allow rapid responsiveness on demand development and volatility to the benefit of stores from shorter lead times on the replenishment cycle and higher delivery frequencies on average. Last mile logistics is often referred to as the last stage of business-to-customer (also known as B2C) package delivery inclined with proximity to customers. It takes place from the order infiltration point (fulfilment centre) to the ultimate consignee's preferred destination point (such as their home), for receipt of the goods. The last mile delivery companies have to achieve cost efficiency and quality customer service while gaining better responsiveness, flexibility and reliability. Last mile (LM) is a phrase utilised within the supply chain network and in the transportation field, intending to depict the transportation of individuals as well as merchandise from a distribution centre point up to the ultimate terminus in the home as the last stretch of a business-to-consumer (B2C) package (Lim and Srai, 2015:86). It involves adapting logistics processes to ensure final delivery is comfortable for customers and efficient. The noteworthy role players engaged in last mile logistics are the retailers, the last mile service providers and the customer. The dependable delivery points, and reliable and flexible transport modes either in-house or third-party logistics service providers include integrated logistics service provision across extended channels (Sighiri, Wilding, Mena and Bourlakis, 2017:61). Having a proper network design and various fulfilment rules built into a fully functional order management e-commerce fulfilment; enterprise resource planning; and/or warehouse management system (WMS) solutions paves the way for a smooth last mile. The order fulfilment model spans order management, WMS and transportation management system (TMS) functionality underpinned by a software-as-a-service (SaaS) to integrate the software with a client's enterprise resource planning (ERP) system, WMS to obtain order and inventory data, and TMS for 3PL/4PL/5PL last mile order fulfilment platform and dynamically optimizing delivery routes.

\section{Figure 1: Last mile: Support Spans Strategic to Tactical}

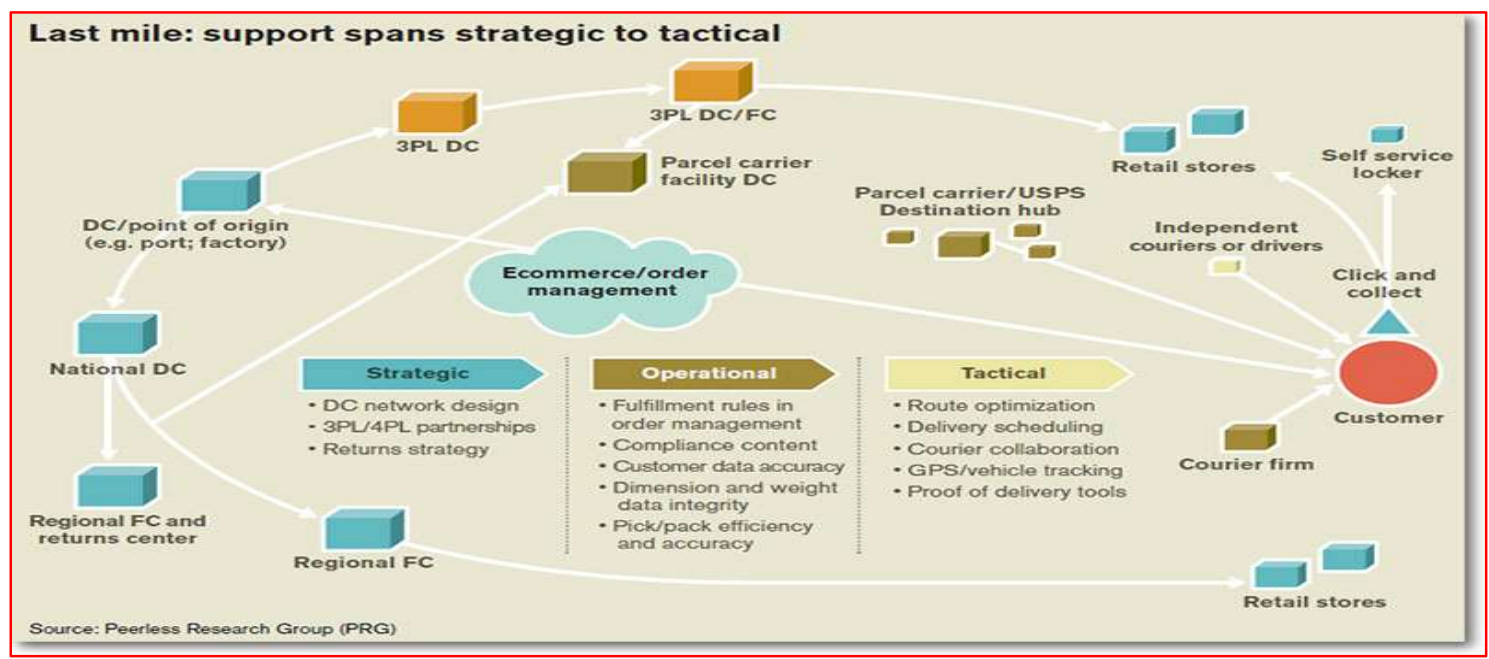

Source: Peerless Research Group 
Effective final delivery starts as far upstream as the structure of the distribution network. Retailers and direct-to-consumer marketplaces need to make decisions about whether they will have traditional DCs complemented by separate fulfilment centres (FCs) geared for picking and packing item-level orders, or whether they will have combined DCs and FCs and determine how much geography each facility should service. These types of network design decisions will drive where the inventory will be stored, which is going to also going to dictate quite a bit about the final delivery that needs to take place.

\section{Theoretical Framework}

The technology-organisation-environment (TOE) framework was developed by Tornatzky and Fleischer (1990). It depicts that the adoption of omni-channel distribution systems within an interrelated distribution network of in-store and online stock is influenced by factors pertaining to the technological, organisational, and internal and external environmental context. This study will employ the principles supported by the Technology Organisation Environment (TOE) framework. The framework describes the factors influencing technology adoption and the propensity to absorb online orders as well as how a firm's adoption and implementation of omni-channel distribution innovations is influenced by the technological, organisational and environmental contexts. According to Lin (2014:80), the TOE framework is a major determinant of the decision to adopt an omni-channel distribution system as it is "enabled by the characteristics of information technology (IT) innovation itself", while the extent of adoption is "driven by organisational readiness, and influenced by environmental factors", especially the situation of suppliers, customers and competitors (Zhu, Dong, Xu and Kraemer, 2006:601). The technological phase, underpinned by the adoption theory, points out that "many innovations do not achieve the expected results for failure to satisfy the requirements of potential adopters (Figueiredo, 2005). It is viewed as omni-channel information technology (IT) adoption that refers to "the adoption of new methods of order placement and fulfilment, integrated information, order and financial process capabilities, and/or proficient distribution network systems, to conduct value chain activities (Liu, Ke, We, Gu and Chen, 2010).

\section{Technology Paradigm}

The technologies of Industry 4.0 affect the connection of products, customers, production, and service companies. Digitisation makes the supply chain solutions more efficient, flexible, and customerfocused. The technological context describes the dependence adoption on the pool of technologies as well as relative network benefits and compatibility, and all the accessible external and internal technologies (Gutierrez, Boukrami and Lumsden, 2015:28). The increased complexity of these interconnected logistics networks needs a sync in design and the flexi-responsive-operation of first mile and last mile supply chains include a huge number of problems: facility location, routing, scheduling, design of loading unit building and packaging processes, budgeting, warehousing, and assignment or queuing. Geschickter, Kutnick, Velosa, Perkins, and Steenstrup (2015:31) argued that businesses need to consider the environment in which they are in and the barriers that may limit the use of the Internet of Things (IoT) to set out plans for businesses to effectively use IoT.

\section{Figure 2: The IoT Roadmap}




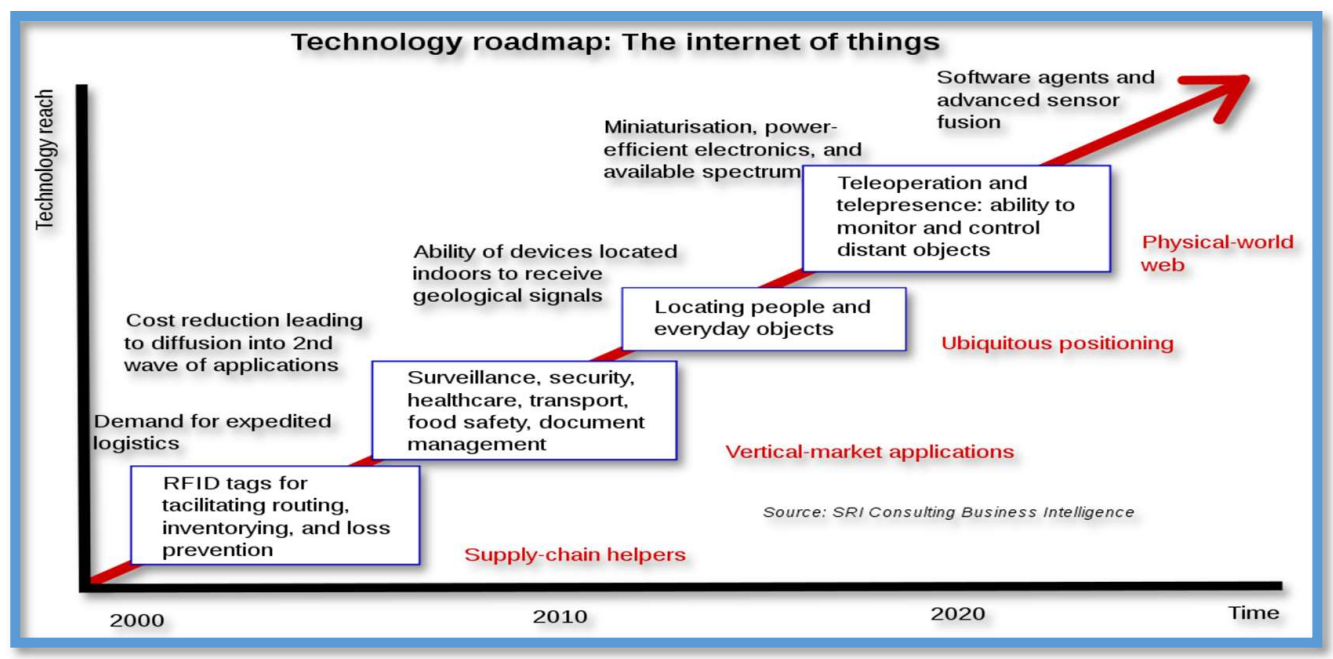

\section{Source: SRI Consulting Business Intelligence (2019)}

Thus, an integration of the Internet of Things technologies into a warehouse management system for an apparel group requires better infrastructure based on several technologies such as radio frequency identification (RFID), Wi-Fi, Bluetooth, sensors, and cloud computing. IoT can be used for monitoring, tracking products, creating an intelligent transportation system, and for demand forecasting, reduced inventory costs as well as the bullwhip effect across the supply chain (Bhattacherjee, 2012:31). According to Liu and Sun (2017:3) the Internet of Things (IoT) refers to internet-connected information communication technologies amongst people and within organisations and throughout different industries including the supply chain. The Internet of Things should bridge digital retail (online) and physical reality (offline - brick and mortar) and powers information-driven automation and improvements on the level of business, society, and people's lives. IoT as a disruptive technology in the business environment, organisations use the technology to leverage opportunities such as connecting devices to create new services in improving customer experience, optimising supply chain operations, and to create new opportunities to generate revenue.

\section{Figure 3: The Internet of Things}

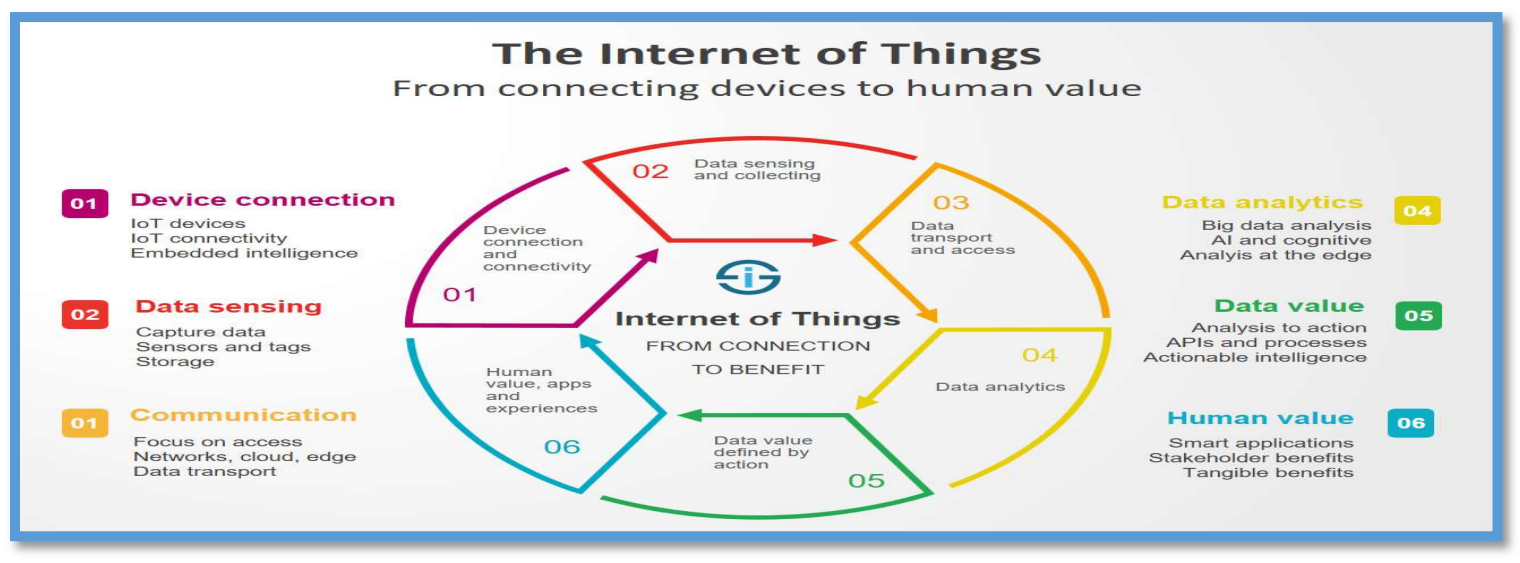

\section{Source: i-Scoop (2020)}

The Internet of Things (IoT) is the digitising of the physical world with more robust data analytics to capture the maximum value of data-driven decision making at lower costs for omni-channels distribution trajectory. IoT is changing how goods are made (3D printing and smart manufacturing) and 
distributed (smart and driverless vehicles), how products are serviced and refined (first and last mile virtual logistics), and how products are monitored from embryotic phase to cradle-to-cradle. Capturing that potential requires innovation in IoT technologies like self-driven vehicles or trucks for swift delivery and a business model catering for last mile efficiency, and investment in omni-channel capabilities.

\section{Environmental Paradigm}

The environmental context relates to facilitating and inhibiting factors in areas of operations (Kurnia, Karnali and Rahim, 2015). An environmental market in which businesses operate include the industry, competitors, access to resources and the dealings with the government (Khosrow-Pour, 2013:62). The most important field of environmental impact-related research is focusing on services through road transport. Research shows that first mile and last mile trips with transit may increase multimodal trip emissions significantly, mitigating potential impact reductions from transit usage (Hoehne and Chester, 2017). Environmental impact can be measured in city logistics solutions; pollution and urbanistic considerations which have led to a change in the use of private vehicles, and the application of new technologies in dense city centres is used more and more often, like accelerating moving walkways (Scarinci, Markov, and Bierlaire, 2017). The last mile logistics has been characterised as the most expensive part of the supply chain, featuring a negative impact on pollution and operation costs. However, in densely populated areas, the number of possible material flow paths is extremely increased, but in this case, there is a great possibility to optimise the last mile solutions (Bányai , Illés, and Bányai, 2018:15). The integration of last mile and first mile solutions increase the complexity of supply chain logistics. Improving the efficiency of FMLM delivery is a major driver for the success of e-commerce (Cardenas, Dewulf, Beckers, Smet, and Vanelslander, 2017). Intelligent retail environment, product enhancement, interconnected supply chain, and data-driven business are the key factors of successful FMLM processes.

\section{Organisation Paradigm}

The organisational context captures the business characteristics such as the scope, size, managerial structure, technology readiness, the quality of human resources and a number of slack resources available in the business (Oliveira and Martins, 2014; Gutierrez, et al., 2015). Organisation stakeholders or Third-party logistics (3PL) providers allow the management to outsource processes of supply chain functions. Parcelninja, as an e-commerce fulfilment specialist, provide scalable outsourced e-commerce warehousing and fulfilment for a small and large online business. Their services can include a wide range of first mile or last mile operations, like warehousing, loading, and unloading, packaging, shipment, and collection (Publow, 2007). Last-mile delivery involves three main entities - customers, merchants, and delivery providers - each with their own set of expectations and challenges. Customers have an increasingly complex set of expectations regarding the speed, flexibility, security, and cost of delivery. Many customers would also like the flexibility to shop in store and have items shipped home or to have the ordered items shipped to a different location, such as an office, a self-service locker, or other pickup points. The increase in express shipments, also caused by the growing sales of the online market, new ICT and the Industry 4.0 paradigm that allow retrieving a huge amount of data generated from infrastructures, devices and vehicles are factors to be managed in a city that has to be rethought in terms of the mobility of goods (Ranieri, Digiesi, Silvestri and Roccotelli, 2018:2). IoT as a disruptive technology in the business environment, organisations use the technology to leverage opportunities such as connecting devices to create new services in improving customer experience, optimising supply chain operations, and to create new opportunities to generate revenue. The identification, traceability and realtime tracking of goods in supply chains have always been difficult, because of the heterogeneity of 
platforms and technologies used by various actors of the chain. The Internet of Things brings a new approach, enabling organisations to collect, and transfer, store and share information on the logistics flow for better cooperation and interoperability between supply chain partners. This logistical collaboration behemoth from an invisible hand of a pull-based marketplace brings the tenet of vertically integrated network that provides inherently optimised advantage and entrenched positioning to dominate both the retail and the transportation sides of the business. Although new delivery approaches such as stores hiring their own delivery personnel and start-up outsourcing delivery vehicles and drivers can operate effectively only on a very local basis, Uber eats, and fast-food outlet-based delivery systems, have gained few advantages by building scale geographically in the apparent industry.

\section{Research Methodology Research Design}

Yin (2014:26) defines a research design as "a rationale that links the research questions to the data collected and conclusions drawn". The purpose of conducting a case study is "to establish a complete picture" of the entire omni-channels retailing and distribution through the examination of a real-life example of an apparel group (Hair, Page, Money and Samoul, 2007:203). The apparel division of the group was used as a case study and data was collected from experienced employees to understand how the Group manages the first mile and last mile customer orders through its distribution network and how virtual technology and IoT assist on fulfilling orders to remain globally competitive. Research philosophy is described as "a system of beliefs and assumptions about developing knowledge" (Saunders, Lewis and Thornhill, 2016:124). Pragmatism is a set of ideas supported by many people, who value subjective and objective knowledge. It was done by taking into consideration, the theory of TOE, concepts of omni-channel operations, retailing, inventory, technology, and logistical distribution (FMLM), and ideas from interviewees and respondents in relation to the phenomenon. The roles of the subjects reflect the instruments of thought and practical results in some contexts (Saunders et al., 2016:143). The criticality of using multiple techniques is recognised by pragmatism philosophy (Creswell, 2014). The pragmatic approach starts the research with a problem of fuzzy omni-channel operations with the objective of contributing to a practical resolution of managing demand-driven order fulfilment frequencies using first mile and last mile that may inform future practice (Saunders et al., 2016:143). Therefore, pragmatisms permit a middle stance (philosophically and methodologically), selecting a mixture of methodologies that are suitable in responding to research questions (Johnson and Onwuegbuzie, 2004). This philosophy acknowledges that reality can be discovered from the perspectives of many people and from different individual perspective of the social world. A cross sectional time horizon was utilised to establish current order fulfilment through omni-channel distribution in the company. The paper used a concurrent mixed methods research design to collect the data, separately analyse them, then compare the outcomes to determine whether the findings are related or not (Creswell, 2014). Omni-channel retailing is a contemporary topic and very little research has been conducted on this phenomenon, especially in a South African context. This exploratory study employed a mixed method approach. The study sites were Durban, the Apparel Group head office, and the distribution centre and extended supply chain as well as the well-integrated third party logistics (3PL) service provider situated in Durban.

\section{Sampling}

Non-probability sampling is commonly used in business research case studies (Lewis et al., 2009:233). Purposive sampling ensures that the research questions are posed to the right people. The sample size for interviews varies based on the scope of the research and the proximity of the interviewer to the participants in the case of face-to-face interviews (Schindler and Cooper, 2008:172). The qualitative part of the paper comprised of 13 Directors and Senior Managers from the Group and the 3PL provider. 
The sample size is dependent on the desired precision which is translated into the confidence level and size, the dispersion of the population, the population size and population homogeneity. Schindler and Cooper (2008:408) state that a 95 per cent confidence level is frequently applied to quantitative research studies and Krejcie and Morgan's (1970:607) sample reference table assumes a standard error of five per cent. The authors highlight that the sample size increases as the population increases at a diminishing rate up to the point of 380. Sekaran (2010:295) endorses this table. Based on a population of 333 managers (322 store managers and 11 area managers) from 161 stores and a standard error of five per cent, 175 Store Managers, and Area Managers were surveyed for the quantitative component of the study (with the response rate of $85 \%$ equals 148 responses). Stratified random sampling was used to decide which position each respondent of the Group belong to, hence maintaining the representative nature of the study. According to Cooper and Schindler (2014:379) and Saunders et al., (2016) stratified random sampling is a process of constraining and modifying a sample to include subjects or elements from each of the segments and is based on several attributes. Stratified sampling specifies the traits to which a sample can be distributed.

\section{Survey Instruments and Administration}

A survey is "a system for collecting data based on the defined objectives with the intention of analysing the results and compiling findings in response to the research question" (Ritter and Sue, 2012:3). The respondents were made aware of the study via e-mail communication. All participants and respondents were required to provide informed consent and were assured that they would remain anonymous. The funnelling approach was applied to gauge the respondents' views on omni-channel retailing, followed by more specific questions relating to the challenges of omni-channels distribution on order fulfilment by the group using FMLM logistic services (Dumay and Qu, 2011:249). The questions were semistructured in a logical sequence following the study's themes in a qualitative strategy. Surveys are "a useful tool to gain information from a large sample in a short space of time; and the survey was selfadministered electronically using a web-based questionnaire via Survey Monkey in a quantitative strategy. However, access to Store Managers, and Area Managers was limited. The respondents had the option of uploading the survey via the store interface, point-of-sale, or via e-mail. Owing to the IT department's resource constraints, the surveys were distributed via e-mail to every store in the sample. The e-mail addresses were acquired from the network teams subsequent to the gatekeeper's permission from the group and ethical clearance being acquired from the University Ethics office. The Human Resources Executive and Omni-channel Manager advised stores of the survey. The interviews were conducted. A hierarchical questioning structure which sequenced the broader questions at the beginning of the interview followed by specific questions, was used to put the participants at ease. Although an audio recording device was used, notes were also taken during the interviews. Greener and Martelli (2015:113) suggest that interviewees be given a copy of the interview questions in advance to facilitate a reflective response.

\section{Data Analysis and Interpretation}

Quantitative and qualitative data were collected to establish how the use of omni-distribution channels are contributing to stock replenishment and in-store order fulfilment, as well as how information systems facilitate an omni-distribution approach using first mile and last mile systems. Whilst the survey enabled an investigation of store operations, the interviews probed further to gain a more extensive understanding of the phenomenon. Abfalter, Muller and Raich (2014:737) distinguish between triangulation, mixed method and the hybrid approach by "identifying the focus of the study, data sets used, analysis methods and degree of integration of the methods". According to Davis and Golicic (2012:727), "a mixed method study combines quantitative and quantitative research approaches of a single study to fully understand phenomena and reduce bias associated with the use of a single 
method". A mixed method approach employs a moderate level of integration at the interpretation and conclusion stages of a study. Abfalter et al. (2014:737) concur with Davis and Golicic (2012) and add that triangulation "seeks to combine different methods with the intention of unpacking complex relationships in the study either through a comparative, convergent, or sequential design". The authors are of the view that a combined approach should utilise intertwined analysis, known as the hybrid approach. Hence, the Statistical Package for the Social Sciences (SPSS) was used for the quantitative and NVIVO for the qualitative analysis. These were performed separately but the interpretation and conclusion were integrated. The quantitative and qualitative methods were conducted sequentially and carried equal weighting in the study. The timing of the methods and the weighting of each method are supported by the purpose of the study. "A sequential study entails the second method being conducted subsequent to the researcher acquiring the results of the first method" (Abfalter et al., 2014:737).

\section{Quantitative Analysis}

The mixed method strategy is adopted to handle and implement the quantitative and qualitative approaches is the concurrent mixed methodology. From epistemological perspectives, objectivity enquiry of positivists believe that empirical methods in quantitative approaches are designed to produce data that are not influenced by humans (Bryman and Bell, 2011:15; Saunders et al., 2016:136). Triangulating the outputs from both the quantitative and qualitative analysis is discussed in this section using a method of triangulation. Their presentation, interpretation and analysis will be explained accordingly.

\section{Frequency Distribution}

Frequency is an account of the times a phenomenon occurs which is represented as a percentage or cumulative percentage. Frequency is used to identify commonality in responses which will be represented graphically using charts. The binomial tests are used to establish whether the observed distribution of a dichotomous variable is the same as what is expected from a specified binomial distribution. By default, each named variable is assumed to have only two values, and the distribution of each named variable is compared to a binomial distribution with $p$ (the proportion of cases expected in the first category) equal to 0.5 . If $p<0.05$, there is a high level of statistical significance and $p<0.025$ for a two tailed test.

The sample comprises 175 respondents of which the response rate is 85 per cent (148 responses). The useable responses comprised 70 per cent strata of middle and senior management. Approximately 68 per cent of the respondents have more than five years managerial experience of which 56 per cent have more than ten years managerial experience. This result indicates that majority of the responses are from a group of experienced managers. Using SPSS, the average of the coefficient of all items is calculated to determine the Cronbach Alpha. Coefficients below 0.7 are considered to be moderate and coefficients of 0.7 and above are considered good (Hair et al., 2007:244). The questions scored 0.945 on the Cronbach Alpha out of 61 number of items implying that the sample is highly reliable. The reliability of statistics constrains the circumlocution of rudimentary knowledge of the magnitude of sales, whether the extension to online buying has increased, decreased, or had no influence on sales. The tentative response indicates that 60 per cent of respondents are of the view that the extension to online buying increased sales, 14 per cent decreased, 22 per cent remained unchanged, and four per cent unspecified. 


\section{Figure 4: Store delivery notification}

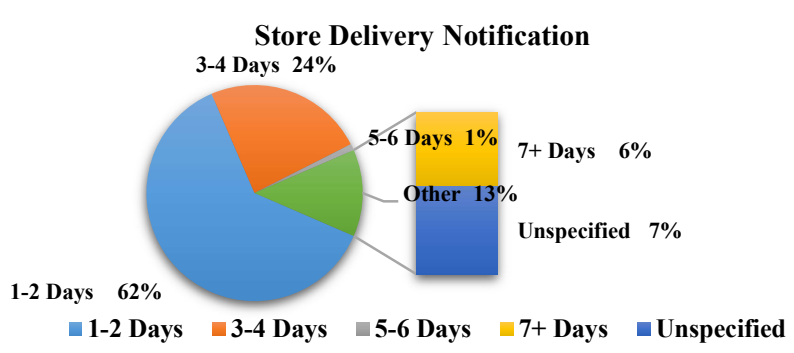

The paper ponders on how many days in advance the branch received notification of the customers' orders to be received by the store. In weighing the options of 1-2 days, 3-4 days, 5-6 days, 7 or more days, the paper divulged that 62 per cent of the respondents indicate that the store receives notification of the orders 1-2 days in advance, whilst 24 per cent reflected that their branch received notification within 3-4 days. The remaining respondents are concerned about receiving communication later, one per cent selected 5-6 days, six per cent selected seven days or more and seven per cent did not dignify the engagement.

\section{Figure 3: Parcel Tracking}

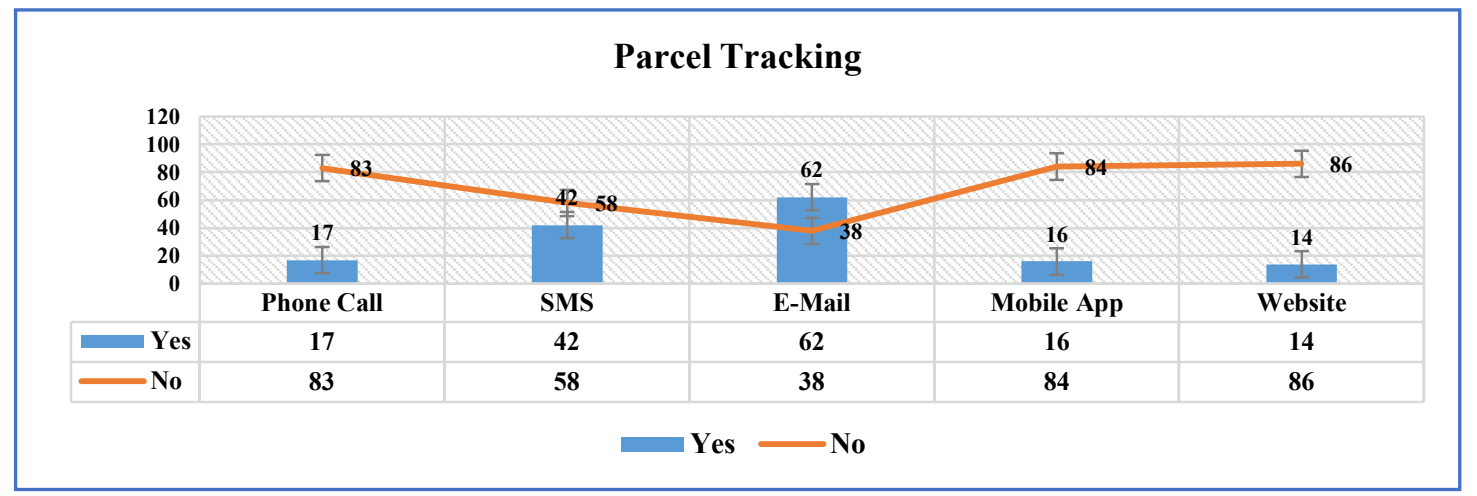

The most commonly used medium of parcel tracking by customers reveals that 62 per cent of the respondents selected email and 42 per cent highlighted SMS as the critical medium by customers. Using the binomial test, a significant proportion indicated that the alternate tracking methods were not used: phone call $(83 \%, p<0.025)$, mobile application $(84 \%, p<0.025)$ and website $(86 \%, p<0.025)$.

\section{Figure 4: Omni-distribution system- Level of Agreement}

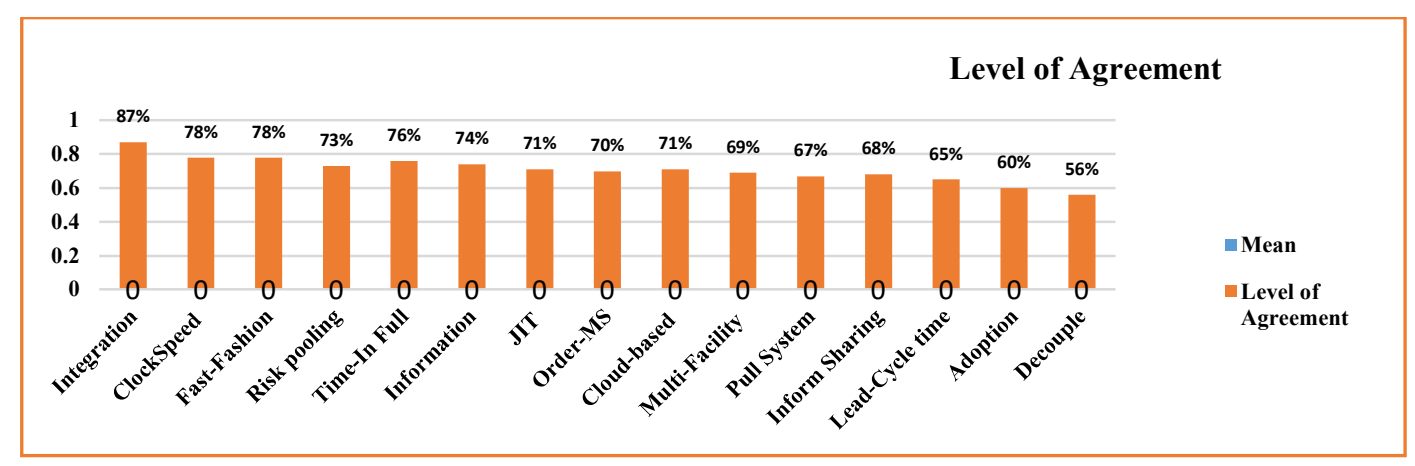


There is a significant level of agreement at $p<0.05$ for all statements. Sixty per cent of the respondents agreed that the adoption of omni-retail channels influences the supply chain retail distribution system to ramp-up the veracity of first and last mile dexterous performance. On the propensity to increase sales $(60 \%)$, the magnitude of customer demand (mean=4.09) influences 'in full' and 'on time' order fulfilment $(76 \%)$ in the overall distribution system. The change in demand influences fulfilment lead time and cycle time $(65 \%)$ in the distribution network, however, when demand is unknown, the supply chain can be decoupled to respond to forecast orders until stock reaches the distribution point, then distributed based on known demand by pull supply chain activities (67\%) using the push-pull system with a decoupling point $(56 \%)$. The integration of both the channels (highest mean=4.45) and the information systems (mean=4.03) improve in full and on time order fulfilment (74\%) where the information sharing and visibility within the virtual distribution network $(68 \%)$ provide an excellent customer experience (87\%). Online channels offer improved product variety to customers. It is essential to note that the omni-channel distribution network enhances the speed and dependability of customer service (78\%), by delivering stock in small frequent loads just-in-time (JIT)to improve response to changes in demand (71\%). It supports last mile order fulfilment through on time delivery and order fill rate.

The fast fashion retailing channels $(78 \%)($ mean $=4.13)$ require agile supply chains with a quick response strategy and shorter cycle times. The omni-channel distribution system aims to pull supply chain activities (67\%) from demand driven orders to reduce system inventory, with cloud-based technology providing visibility of detailed data in real-time from various sources across the chain $(71 \%)$. The utilisation of a main distribution centre and multiple smaller distribution facilities (69\%) benefits mass last mile distribution in two ways: firstly it reduces the risk with online channels due to risk pooling (73\%) from broader product assortment to customers; and secondly, an order management system (70\%) unifies order processing across the retailer's network of physical stores.

\section{Qualitative Analysis}

In the subjective enquiry, the pragmatists suggested that reality is dynamic and that the best way people can learn is to apply knowledge acquired through thoughts and experiences. These meanings are investigated by the interpretivists that postulate human behaviour and the insight to human behaviour is dependent on their varied experiences and interpretations to life (Saunders et al., 2016:140). Thematic analysis is the first qualitative method of analysis that researchers should learn, as it provides core skills that are useful for conducting many other forms of qualitative analysis. It is "a flexible and useful research tool, which can potentially provide a rich and detailed, yet complex account of data" (Braun and Clarke, 2013:78). A thematic map refers to the graphical presentation of themes, categories, and their relationships, involving a thorough explanation of each theme, their criteria and categories. It helps to outline the objectives that were achieved from the interviews (Ranney et al., 2015:22). The categorisation and summation of data are utilised to explore the contribution of each department towards fulfilling customers' orders on time and in full. The interviews were transcribed from the audio recordings, categorised and summarised thereafter based on the research objectives. Thematic analysis followed the necessary phase, identifying, analysing, and reporting themes within the data. It was important to become familiar with the data for accurate translation and transcription by understanding the meanings, the transcripts and audio recordings and notes taken to import into the software, the development of themes, aggregating the categories and the emergence of themes from categories. This study developed the following themes and categories that interrogates the study objectives. 
Figure 5: Thematic Mapping

\begin{tabular}{|c|c|c|c|}
\hline $\begin{array}{c}\text { THEMIES } \\
\text { Replenishment }\end{array}$ & $\begin{array}{c}\text { CATEGORY } \\
\text { Order Fulfilment }\end{array}$ & $\begin{array}{c}\text { CODES } \\
\text { In full } \\
\text { On time }\end{array}$ & $\begin{array}{l}\text { To what extent does the last mile for demand-driven store } \\
\text { replenishment influence the level of retailer's order fulfilment } \\
\text { (in full and on time) }\end{array}$ \\
\hline $\begin{array}{c}\text { Integration } \\
\text { Strategy }\end{array}$ & $\begin{array}{c}\text { Inbound Activity } \\
\text { Outbound Activity }\end{array}$ & $\begin{array}{c}\text { First mile } \\
\text { Door to door } \\
\text { Click and Collect } \\
\text { Overnight }\end{array}$ & $\begin{array}{l}\text { How does the group integrate the inbound and outbound } \\
\text { activities to manage the first mile, door to door, click and } \\
\text { collect, and overnight store fulfilment? }\end{array}$ \\
\hline $\begin{array}{c}\text { Order and } \\
\text { Stock Accuracy }\end{array}$ & $\begin{array}{c}\text { Order accuracy and fill } \\
\text { rate }\end{array}$ & $\begin{array}{c}\text { Online and Offline } \\
\text { Initiatives }\end{array}$ & $\begin{array}{l}\text { What are the initiatives in place to improve order accuracy } \\
\text { and fill rate? }\end{array}$ \\
\hline $\begin{array}{c}\text { Integrated } \\
\text { Information } \\
\text { Systems }\end{array}$ & $\begin{array}{c}\text { Information Sharing } \\
\text { SC Visibility }\end{array}$ & $\begin{array}{c}\text { Virtual Omni- } \\
\text { distribution }\end{array}$ & $\begin{array}{l}\text { What is the contribution of integrated information systems } \\
\text { towards information sharing and visibility within the virtual } \\
\text { omni-distribution network? }\end{array}$ \\
\hline
\end{tabular}

Theme One: To what extent does the last mile for demand-driven store replenishment influence the level of retailer's order fulfilment (in full and on time)?

The value-based fast and new fashion business uses website and mobile application catalogue items for the online store and luxury of store visits and advertising for brick-and-mortar stores to bring volatility to on demand changes. The level of visibility of inventory and orders, and the service level agreement between the retailer and 3PL has an implication on the last mile for store replenishment and order fulfilment. The allocation to the online store and fulfilment store is based on demand and the cost of transportation, in terms of reducing courier cost and lead days of delivery from store through close-tosource deliveries. The first mile presides optimised operations systems, if the picker cannot locate the item, the order is picked failed and is redirected to the online store. The operations process facilitates more control of inventory through the use of the online store whilst achieving reduced cost, effective use of stock, reduced distribution cost to customer and enhanced speed to market through the use of fulfilment stores. The online and fulfilment store customise operational processes system-based on route optimisation. Couriers have specific pick-up times for stock delivery to the hub based on the planned sorting time, loading, vehicle types and on expected volume to synchronise the omni-channels' first mile and last mile operations process designs.

Theme Two: How does the group integrate the inbound and outbound activities to manage the first mile, door to door, click and collect, and overnight store fulfilment?

First Mile on Online and Offline: The online facility expects a delivery between 8-10 in the morning to manage inbound and outbound. The integrated systems provide visibility on expected stock delivery through the advanced shipping notice, and the shared information is received by the facility two days in advance to build on capacity and resources such as labour planning. On the first mile, the layout, height and categories of the product within the facility are grouped as the layout influences the pick rate. The online store has smaller single item locations where the boxes are open and easily accessible in contrast to being stacked. By having the stock well laid out, less time is spent looking for the item, which speeds up the pick. The online store has adapted their system for business to consumer fulfilment which requires fine picking in comparison to full case picking. On the last mile, the in-house logistics management system (LMS) is integrated with many preselected couriers based on courier cost optimisation using a predefined algorithm to select the cheapest courier that can deliver the order the fastest.

Door to door Store Fulfilment: Door to door orders are prioritised first, followed by store economy and store express. Door to door orders are collected from the fulfilment store at 12pm, and store 
economy and store express orders are collected at $4 \mathrm{pm}$. If the courier misses the time to pick up, the order overlaps to the next day morning and delivered to the designated collection store. There are designated click and collect express queues for the customer to collect their order in-store. The online store picks and packs within 24 hours and can achieve same day delivery for areas with close proximity to the facility. The fulfilment store plays a similar role to the online store but is able to provide sameday or next day delivery in the region the store is situated.

Click and Collect: Different organisations have different key drivers for click and collect. In some organisations high availability is a key driver whilst in other organisations higher service level is a driver. Changing the availability of the courier company has an implication on cost and quality. There is a trade-off between low volume and high variety processes versus high volume and low variety processes. From a routing perspective couriers provide additional services to identify the cartons so that it gets some form of priority using track and trace. The form of prioritisation used is dependent on the cost of the service.

Store Overnight Delivery: Overnight deliveries may be nominated compared to a delivery with a three-day lead time. The option with the 3-day lead time may utilise the existing network whilst the overnight delivery may require a specialised delivery vehicle which is out of the usual distribution network. The overnight delivery can be done at a higher price point compared to the three-day delivery. In the current model for store economy, the courier is meeting a price point. Cartons loaded at the courier loading store economy orders follows FIFO. Deliveries via post office are cost effective with longer lead times as compared to deliveries via economy.

Last mile deliveries via door to door and store express. The online store has a system that optimises door to door, and door to store deliveries using predefined algorithms in the logistics management system. The system selects the most cost effect courier however, the rate is still at premium compared to store economy deliveries. The 3PL developed a track and trace software like the Uber model whereby there is visibility of the parcel and delivery vehicle. Other couriers contracted with them also use similar applications to gain visibility and achieve route optimisation. The store fulfilment is being pivotal in achieving on time delivery with the objective of omni-channel intents exceeding the customer's expectations.

Theme Three: What are the first mile and last mile initiatives in place to improve order accuracy and fill rate?

Omni-channel retailing exposes stock accuracy as online orders need to be fulfilled at SKU level. Stock inaccuracies are detected at stores during scheduled stock takes whilst fulfilment stores reconcile inventory on a daily basis. The online and fulfilment store is set to 100 per cent audit in the DC contrast to blind receiving for traditional brick-and-mortar to ensure the stock visibility is 100 per cent accurate. The system at the fulfilment store lets the user view the code and the picture of the stock and rejects incorrect items that were scanned. The online store performs a pre-scan, followed by a detailed scan whereby every item received is scanned and the stock on hand is verified every day. All defective or excess stock is sent to the returns DC to be flushed out to the clear-out store. If orders cannot be fulfilled by the fulfilment store, the orders are redirected to the online store. The customer service contacts the customer to fulfil the order without the item. Most inaccuracies occur due to the electronic finance technology (EFT) process in comparison to payment via credit card as a result of the delay in using EFT. The respondent added that more integration is needed for EFT as every step in the process needs to be integrated for successful omni-channel execution. 
Theme Four: What is the contribution of integrated information systems towards information sharing and visibility of first and last mile within the virtual omni-distribution network?

The supply chain uses a single system comprising multiple sub-systems which are linked from the point of origin (PO) creation until stock is delivered to the store however, the subsystems are not integrated in real time, nor is there visibility of the last mile. An integrated system such as a Distribution Order Management system (DOM) backward integrates demand and allocates the best place that demand should be fulfilled based on location to customer proximity which is channelled through the system. The e-commerce system which is also linked to the host system receives historic information concerning each touch point in the order process however, the data is not in real time. The e-commerce team uses a web order dashboard which provides visibility of the courier, tracking number, payment method. However, there is no visibility of the last mile, between the order being shipped and delivered and as a result there is no end to end visibility of every touch point. The transport service provider recommended a single customer management process as the retailer and logistics provider are both running call centres to assist with customer queries. Currently, orders cannot be split for fulfilment to occur from different sources. The business still needs to develop the omni-distribution model.

\section{Discussion of Results}

The adoption of omni-retail channels has influenced the extension to online buying and the supply chain retail distribution system from increasing sales to ramping-up the veracity of first and last mile dexterous performance. On the propensity to increase sales, the magnitude of customer demand influences 'in full' and 'on time' order fulfilment in the overall distribution system. The branch received notification of the quantity of customers' orders to be received by the store in 1-2 days. The most commonly used medium of parcel tracking by customers is email while the SMS was also highlighted as a critical medium by customers.

Increasing urbanisation is making the last mile of delivery more complex and critical for the success of e-commerce companies to deliver with even more speed and convenience. Companies that can improve performance in the areas of flexible transport networks, automation and data will be better positioned to compete. Localisation (shifting supply chain to focus more on regional fulfilment to shorten last mile), and flexi-delivery (build flexibility into the networks) have been facilitated by innovative technologies by increasingly adopting virtual reality, artificial intelligence, Internet of Things, cloud computing and the use of big data analytics to work towards more flexible models. The integration of both the channels and the information systems improves in full and on time order fulfilment in store and online. The information sharing and visibility within the virtual distribution network provide an excellent customer experience. However, there is no visibility of the last mile, between the order being shipped and delivered, and as a result there is no end-to-end visibility of every touch point. Virtual reality is applicable for the system at the fulfilment store where the user views the code and the picture of the stock and rejects incorrect items that were scanned. Nevertheless, the propensity to use the big data analytics and artificial intelligence on the online store can perform a pre-scan, followed by a detailed scan whereby every item received is scanned and the stock on hand is verified every day. The last mile system tracing and tracking with multisensory modes tentatively providing visibility for intransit trucks and last mile operations. The first mile and last mile logistic services with the online and fulfilment store set on 100 per cent audit in the DC in contrast to blind receiving for traditional brickand-mortar ensures the stock visibility at 100 per cent accuracy with IoT, big data analytics, driverless vehicles, and AI technologies.

The better coordination and more accurately matched demand for the delivery services build a last mile exchange platform that drives delivery decisions, allows retailers and logistics supply chain partners the 
collaboration to sync in better delivery demand, and to entrench agile, responsive, and continuous adjustment and flexibility to the inherent oscillation and variability of the last mile. The omni-channel distribution system aims to pull supply chain activities from demand driven orders to reduce system inventory, with cloud-based technology providing visibility of detailed data in real-time from various sources across the chain. The store fulfilment is pivotal in achieving on time delivery with the objective of omni-channel intents exceeding the customer's expectations. The difficulties of managing demand on a given day, which is especially evident at peak times such as Black Friday and holiday seasons, are built into the current e-commerce ecosystem. Essentially, the omni-channel distribution network enhances the speed and dependability of customer service, by delivering stock in small frequent loads JIT to improve response to changes in demand. It supports last mile order fulfilment through on time delivery and order fill rate. Last mile couriers have specific pick-up times for stock delivery to the hub based on the planned sorting time, loading, vehicle types and on expected volume to synchronise the omni-channels first mile and last mile operations process designs. On the last mile, the in-house logistics management system (LMS) is integrated with many preselected couriers based on courier cost optimisation using a predefined algorithm to select the cheapest courier that can deliver the order the fastest.

The extended supply chain delivery network has complex big data requiring data analytics to yield improved convenience, transparency, efficiency and cost savings. Technological and analytical omnichannel capabilities for efficient last mile exchange allows a dynamic pricing model open to multiple retailers, targeting a host of customers and bids on by compliment of carriers such as $4^{\text {th }}$ PL, $5^{\text {th }}$ PL and integrated logistics service providers. The 3PL developed a track and trace software like the Uber model whereby there is visibility of the parcel and delivery vehicle. Other couriers contracted with them also use similar applications to gain visibility and achieve route optimisation. The level of visibility of inventory and orders, and the service level agreement between the retailer and 3PL has an implication on the last mile for store replenishment and order fulfilment. The allocation to the online store and fulfilment store is based on demand and the cost of transportation, in terms of reducing courier cost and lead days of delivery from store through close-to-source deliveries. More integration is needed for EFT as every step in the process needs to be integrated for successful omni-channel execution. Door to door orders are prioritised first, followed by store economy and store express. If the courier misses the time to pick up, the order overlaps to the next morning and delivered to the designated collection store. There are designated click and collect express queues for the customer to collect their order in-store. Different organisations have different key drivers for click and collect. In some organisations high availability is a key driver whilst in other organisations a higher service level is a driver. Changing the availability of the courier company has an implication on cost and quality. The overnight delivery can be done at a higher price point compared to the three-day delivery. Deliveries via post office are cost effective with longer lead times as compared to deliveries via economy. The fast fashion retailing channels require agile supply chains with a quick response strategy and shorter cycle times

\section{Reliability and Validity of Instruments}

Measurement tools are considered to be reliable, and valid when they exhibit precise measurement procedures and are characterised as being fit for purpose (Schindler et al. 2008:289). Reliability can be measured using test-retest, split-half reliability, and Cronbach Alpha for quantitative data (Hair et al., 2007:242). The Cronbach Alpha (0.945 out of 61 items) is used to test reliability, as the level of homogeneity of the questions in the instrument is tested scientifically. Thomas (2010:318) elaborates that quality is reflected by the trustworthiness of the research through credibility, transferability, dependability, and confirmability. The credibility of the research is the extent to which its findings match reality. It establishes if there is a match between the constructed realities of respondents 
and those represented by the researcher. Credibility validates whether the findings denote "credible information that have been drawn from the participants' original data and if the interpretation of the participants' original views are correct" (Korstjens and Moser, 2018:121). The strategies which are used to ensure credibility are triangulation, prolonged engagement, member check and persistent observation. It establishes if there is a match between the constructed realities of respondents and those represented by the researcher (Ghauri, Penz and Sinkovics, 2008:699). Transferability is the extent to which the findings can be generalised. Although this is considered a challenge in qualitative data analysis due to its subjective nature, justification of the methodological approach and a detailed description of critical processes and procedures and associated meanings with phenomena (Thomas, 2010:320). A detailed breakdown of the interview process, transcription of audio recorded interviews and thematic analysis justifies the transferability of the research. The instrument is dependable when the steps followed during the course of a research study can be verified through examination and reduction of data. The transparency and a detailed record of interviews facilitates future corroboration of the results. Reliability of the qualitative instrument is also assessed through the similarity of words and phrases using category reliability or inter-judge reliability (Sekaran, 2010:384). Confirmability involves corroboration of the findings by personnel such as auditors or others doing similar research as well as through triangulation. It is achieved through a methodological account of how the research was conducted and by archiving data for future investigation (Thomas, 2010:322). The use of multi-method research promotes the credibility, dependability and confirmability of the overall research findings, ensuring the data is trustworthy and reliable.

It is measured by means of content validity, construct validity and criterion validity. Content validity requires a sample of experts to be consulted to assess the suitability of the items representing a construct on the instrument (Schindler and Cooper, 2008:290). This study was based on established supply chain concepts; hence content validity was ensured by consulting with supply chain experts and academics for the quantitative and qualitative data collection. Content validity of the survey instrument was also achieved by reviewing the relevant literature. In addition, the correspondence with the respondents, as well as the survey documents, were approved by supply chain experts such as the regional omni-channel manager and the omni-channel director, prior to distribution. The survey was also piloted by sending it to ten stores in the Durban region. The feedback was that supervisors and store associates would not cope with the level of the questions and that it should rather be administered to store management and area managers. The survey was subsequently distributed to store managers and area managers in Durban. In addition, triangulation can be used to test the validity of different sources in qualitative data. This helps to provide an inclusive insight into the phenomenon under study. Triangulation allows the researcher to support the results that relate to the phenomenon. The researcher will build a coherent justification for themes that emanated from several sources of data from the participants' perspectives. A combination of both research methods, as well the use of questionnaires and interviews during data collection will enhance the triangulation technique.

\section{Conclusion and Future Study}

The findings reflect that a multitude of fulfilment centre and fulfilment channels are being used to fulfil orders in the retail group omni-distribution model. The current orders fulfilment process aligned with the last mile is effective on the basis of the fill rate, lead time and condition of orders delivered via store economy and store express. However, the door-to-door shipping method and delivery via the postal service for local orders as stores are not exposed to these shipping methods. Future studies should attempt to establish the extent to which orders are being fulfilled via door to door and post relative to delivery via store express and store economy in South Africa, as well as the services offered internationally. Logistics network designs are diverse, customised and locally optimised to transform 
the supply chain into decentralised fulfilment systems to enhance cycle time operations, shorten lead time and shorten the throughput rate on the last mile. A single delay forms a common perception about the business which later flutters in the market in the form of negative reviews and preferring competition. Electronic commerce (e-commerce) businesses have to optimise the e-commerce delivery applications (apps) to keep customers continuously happy as the online retailing market is expected to grow boundlessly with an increasing number of orders being placed by the customers. The modern customers are ready to pay extra for same-day delivery. E-commerce players are spending bundles on new tools and technologies such as IoT, big data, drones, electrical vehicles, GPS, and much more. The smart tracking system increases visibility into the supply chain and the customers can view the order status at every step of the way. A last-mile delivery exchange that connects consumers, retailers, and transportation companies via a digital platform, further challenges the e-commerce ecosystem to produce benefits for consumers, retailers, and the package delivery providers, yielding improved convenience, transparency, efficiency, and cost savings. Such an exchange would create a path forward through the disruption caused by increasing consumer expectations, advances in technology, the emergence of new entrants into the marketplace, and the rise of the sharing economy (PwC report, 2016). The managers further cogitate on flipping the thinking back towards the customer and relying on sales forecasts with long lead times and extended supply chains. Omnichannel distribution on the underlying demand-driven model requires them to ponder about how best to manage a supply chain that is serving a volatile market, in levels of complexity, while the last mile is traced on long distances and lead times from end-to-end logistical specificity and speed.

\section{REFERENCES:}

Abfalter, D., Muller, J., and Raich, M. 2014. Hybrid analysis of textual data. Management Decision, 52(4): 737-754.

Bányai,T., Illés, B, and Bányai, A. 2018. Smart Scheduling: An Integrated First Mile and Last Mile Supply Approach. Hindawi Complexity Volume 2018, Article ID 5180156, 15 pages https://doi.org/10.1155/2018/5180156

Beck, N. and Rygl, D. 2015. Categorisation of multiple channel retailing in Multi-, Cross-, and Omnichannel Retailing for retailers and retailing. Journal of Retailing and Consumer Services, 27:170-178.

Bhattacherjee, A. 2012. Social science research: Principles, methods, and practices $2^{\text {nd }}$ Ed. United Kingdom: John Wiley \& Sons Ltd.

Boateng, D. 2014. An executive compendium of supply chain management terms. Lily Hattingh, $1^{\text {sted. }}$

Braun, V., and V. Clarke 2013. Teaching thematic analysis: Overcoming challenges and developing strategies for effective learning. The Psychologist ISSN 0952-8229: 120-123.

Bryman, A. and Bell, E. (2011). Business research methods. Oxford: Oxford University Press.

Cardenas, D., Dewulf, W., Beckers, J., Smet, and Vanelslander, T. 2017. The e-commerce parcel delivery market and the implications of home B2C deliveries vs pick-up points, International Journal of Transport Economics, 44(2), pp.235-256.

Cooper, D.R. and Schindler, P.S. 2014. Business Research Methods. $12^{\text {th }}$ edition. McGraw-Hill/Irwin, New York, United States of America.

Creswell, J.W. and Creswell, J.D. 2018. Research Design: Qualitative, Quantitative and Mixed Methods Approaches. $5^{\text {th }}$ edition. New York: Sage Publications, Inc.

Creswell, J.W. 2014. Research Design: Qualitative, Quantitative and Mixed Methods Approaches. $4^{\text {th }}$ edition. New York: Sage Publications, Inc. 
Davis, D.F., and Golicic, S.L. 2012. Implementing mixed methods research in supply chain management. International Journal of Physical Distribution and Logistics Management, 42(8/9): 726-741.

Dumay, Q.J., and Qu, S. 2011. The qualitative research interview. Qualitative Research in Accounting and Management, 8(3): 238-264.

Figueiredo, L.A. 2005. A industria de prestacao de servicos logisticos e o mdelo de negocio ASP: Perspectivas elendencias no mercaso brasileiro, Doctoral thesis, UFSC, Folorianopolis.

Geschickter, C.M.C., Kutnick, D., Velosa, A., Perkins, E. and Steenstrup, K. 2015. Predicts and Charting the path to IoT business value. Stamford: Gartner.

Ghauri, P.N., Penz, E., and Sinkovics, R.R. 2008. Enhancing the trustworthiness of qualitative research in international business. International Marketing and Management, 48(6): 689-714.

Greener, S., and Martelli, J. 2015. An Introduction to Business Research Methods. $2^{\text {nd }}$ ed. [Online]. Available at: http://bookboon.com/en/introduction-to-research-methods-ebook [Accessed on 24 March 2016].

Gutierrez, A., Boukrami, E., and Lumsden, R. 2015. Technological, organisational and environmental factors influencing managers' decision to adopt cloud computing in the UK. Journal of Enterprise Information Management. 28. 10.1108/JEIM-01-2015-0001

Hair, F.J., Page, M., Money, A.H., and Samoul, P. 2007. Research methods for business. $1^{\text {st }}$ ed. England: John Wiley and Sons Ltd.

Hoehne, C.G. and Chester, M.V. 2017. Greenhouse gas and air quality effects of auto first-last mile use with transit. Transportation Research Part D: Transport and Environment, 53: 306-320.

Hubner, A., Holzapfel, A. and Kuhn, H. 2016. Distribution systems in omni-channel retailing. Business Research, 9: 255-296

Huré E., Picot-Coupey, K. and Ackermann, C. 2017. Understanding omni-channel shopping value: A mixed-method study. Journal of Retailing and Consumer Services, 39: 314-330.

i-Scoop (2020). The Internet of Things (IoT) - essential IoT business guide. (Online) Available at: https://www.i-scoop.eu/internet-of-things-guide/

Johnson, B. and Onwuegbuzie, A. 2004. Mixed Methods Research: A Research paradigm Whose

Time has Come. American Educational Research Association, 33(7): 14-26. URL:

https://www.jstor.org/stable/3700093.

Khosrow-Pour, M. 2013. E-Commerce for Organisational Development and Competitive Advantage.

Hershey: IGI Global.

Korstjens, I. and Moser, A., 2018. Series: Practical guidance to qualitative research. Part 4: trustworthiness and publishing. European Journal of General Practice, 24(1).

Krejcie, R.V., and Morgan, D.W. 1970. Determining the sample size for research activities. The Educational and psychological measurement, 30(1): 607-610.

Kurnia, S., Karnali, R.J., and Rahim, M.M. 2015. A qualitative study of business-to-business electronic commerce adoption within the Indonesian grocery industry: A multi-theory perspective. Information and Management, 52: 518-538.

Lewis, P., Saunders, M., and Thornhill, A. 2009. Research methods for business students. $5^{\text {th }}$ ed. England: Pearson Education Limited.

Lim, S.F.W.T. and Srai, J.S. 2015. Innovations and Strategies for Logistics and Supply Chains. Ecommerce Last-mile Supply Network Configuration and Logistics Capabilities. Hamburg: epubli (Online) Available at: https://www.researchgate.net/publication/282355706.

Lin, H. 2014. Understanding the determinants of electronic supply chain management system adoption: Using the technology-organisation-environment framework. Technological Forecasting and Social Change, 86: 80-92.

Liu, H., Ke, W., We, K.K., Gu, J., and Chen, H. 2010. The role of institutional pressures and organisational culture in the firm's intention to adopt Internet-enabled supply chain management systems. Journal of Operations Management, 28(5): 375-384.

Liu, M. and Sun.A. 2017. Intelligent assembly system for mechanical products and key technology based on internet of things. J. Intell. Manuf., 28 (2): 271-299 
McBeath, L. 2014. Africa...In an Omni World. Omni Retailing Markets Association (IORMA). [Online]. Available at: http://www.iorma.com/reports/uk-retail-market-opportunityreport-may-2014 [Accessed on 1 May 2014].

Oliveira, T., and Martins, M.F. 2011. Literature review of information technology adoption models at firm level. Electronic Journal of Information Systems Evaluation, 14: 110.

Onstein, A.T.C., Tavasszy, L.A. and van Damme, D.A. 2019. Factors determining distribution structure decisions in logistics: a literature review and research agenda. Transport Reviews, 39:2, 243260. DOI: $10.1080 / 01441647.2018 .1459929$.

op de Woerd, M. (2017, July 3). Hoe Flevoland modereus Zara als eerste uit Spanje lokte [How Flevoland Drove Fashion Giant Zara out of Spain first]. Trouw. (Online) Available at: https://www.trouw.nl/

Piotrowicz, W. and Cuthbertson, R. 2014. Introduction to the Special Issue Information Technology in Retail: Toward Omni-channel Retailing. International Journal of Electronic Commerce, 18(4): $5-15$.

Publow, F. 2007. Consider third party logistics to address your company's challenges, Canadian Apparel, 31, 3:8-9+37.

PwC Report. 2016. Shifting Patterns: The Future of the Logistics Industry. Price Water Cooper.

Ranieri L., Digiesi, S., Silvestri, B. and Roccotelli, M. 2018. A Review of Last Mile Logistics Innovations in an Externalities Cost Reduction Vision. Sustainability. 10, 782:1-18., doi:3390/su10030782.

Ranney, M.L., Z.F. Meisel, E.K., Choo, A.C. Garro, C. Sasson, and G.K. Morrow Guthrie 2015. Interview-Based Qualitative research in emergency care part two: Data collection and results reporting. Academic Emergency Medicine 22,9: 1103-1112.

Ritter, L. A., and Sue, V. M. 2012. Conducting online surveys. $2^{\text {nd }}$ ed. New York: Sage publications.

Saghiri, S., Wilding, R., Mena, C and Bourlakis, M. 2017. Toward a three-dimensional framework for omni-channel. Journal of Business Research, 77:53-67

Saunders, M., Lewis, P., and Thornhill, A. 2016. Research Methods for Business Students. $7^{\text {th }}$ Edition. Harlow, England: Pearson.

Scarinci, R., Markov, I and Bierlaire, M. 2017. Network design of a transport system based on accelerating moving walkways. Transportation Research Part C: Emerging Technologies, 80: $310-328$.

Schindler, P.S., and Cooper, D.R. 2008. Business research methods. $1^{\text {st }}$ edition. New York: McGrawHill.

Sekaran, U. 2010. Research Methods for Business: A skill building approach. $5^{\text {th }}$ ed. New York: John Wiley \& Sons, Inc.

Sekaran, U. and Bougie, R. 2011. Research Methods for Business: A Skill-Building Approach. $5^{\text {th }}$ edition. Wiley Publication.

SRI Consulting Business Intelligence. 2019. IoT Technology roadmap. NOVATEC, June, 2019.

Tate, M., Hope, B., and Coker, B. 2007. The Buywell way: Seven essential practices of a highly successful multi-channel e-tailer. Australasian Journal of Information Systems, 12(2): 147163.

Thomas, S. 2017. Logistics: the backbone of South Africa's retail boom. Pargo. (Online) Available from: https://pargo.co.za/blog/logistics-the-backbone-of-south-africas/

Tornatzky, L.G., and Fleischer, M. 1990. The Processes of Technological Innovation. Massachusetts, Lexington: Lexington Books.

Verhoef, P.C., Kannan, P.K. and Inman, J.J. 2015. From Multi-Channel Retailing to Omni-channel Retailing Introduction to the Special Issue on Multi-Channel Retailing. Journal of Retailing, 91(2): 174-181.

Yin, R.K. 2014. Case study research. Design and Methods. $5^{\text {th }}$ Edition. Los Angeles: Sage Publications.

Zhu, K., Dong, S., Xu, S.X., and Kraemer, K.L. 2006. Innovation diffusion in global contexts: Determinants of post-adoption digital transformation of European companies. European Journal of Information Systems, 15: 601-616. 
